\begin{tabular}{|c|l|}
\hline Title & Random dynamics from a time series of physiological rhythms \\
\hline Author(s) & Sato, Y uzuru; Matsumoto, Kazuhiro \\
\hline Citation & Hokkaido University Preprint Series in Mathematics, 1012, [1] \\
\hline Issue Date & 2012-7-3 \\
\hline DOI & 10.14943/84158 \\
\hline Doc URL & http://hdl.handle.net/2115/69817 \\
\hline Type & bulletin (article) \\
\hline File Information & pre1012.pdf \\
\hline
\end{tabular}

Instructions for use 


\title{
Random dynamics from a time series of physiological rhythms
}

\author{
Yuzuru Sato ${ }^{\dagger}$ and Kazuhiro Matsumoto \\ $\dagger$ RIES / Department of Mathematics, Hokkaido University \\ Kita 12 Nishi 6, Kita-ku, Sapporo, Hokkaido 060-0812, Japan \\ $\ddagger$ Research \& Development Section, Yamaha Motor Co., Ltd. \\ 2500 Shingai, Iwata, Shizuoka 438-8501, Japan \\ Email: ysato@math.sci.hokudai.ac.jp, matsumotokazuhi@yamaha-motor.co.jp
}

\begin{abstract}
A random dynamics with two stochastic terms is modeled based on a time series of physiological experimental data to study synchrony between human heartbeats and pedaling rhythms modulated by music. We investigate reproduced time series, rotation numbers, and invariant densities in the model to explain transitory stagnation motion of synchrony in the experiments.
\end{abstract}

\section{Introduction}

It is common to model biological systems as oscillatory systems in order to extract characteristic rhythms from experimental data $[1,2,3]$. Schäfer et al. address the interaction between cardiovascular and respiratory systems in humans [4]. They report that there are synchronous regimes of different orders $n: m$ and transitions between them under free running conditions. As well, there are some studies on interactions between heartbeats and music under resting conditions [5] and interactions between cardiac and locomotor rhythms under running and cycling conditions [6]. In [6], subjects performed exercises at a high exercise intensity (heart rate: $150 \mathrm{bpm}$ ) because they focused on difference of cardiac-locomotor synchronization between exercise modes (running and cycling). It is, however, pointed out that destructive stimuli such as music become less effective at higher exercise intensities[7, 8].

In this paper, we consider the effect of music from the viewpoint of the interaction between human heartbeats and pedaling rhythms under cycling conditions with moderate exercise intensities. These experiments are motivated by a product development at YAMAHA Motor Co., Ltd. for controlling human physiological rhythms by a music player. A random dynamics with two stochastic terms is given based on a time series of a physiological experimental data (see the method in [9]). We observe time series, rotation number and invariant density by model simulation to explain transitory stagnation motion of synchrony in the experiments.

\section{Experiments}

We performed non-invasive examinations with healthy volunteers (the data we analyzed is generated by a volunteer who is male and 29 years). The subject carried out pedaling exercises in a laboratory with and without listening a music (Rêverie, Debussy, a classic music). The bicycle used in the experiment was fixed by a bicycle trainer (RealPower, Elite). We set the load of the trainer at a middle degree (7\% grade) and its running distance at $1 \mathrm{~km}$ in each condition in order to set the exercise intensity to be medium for the subject.
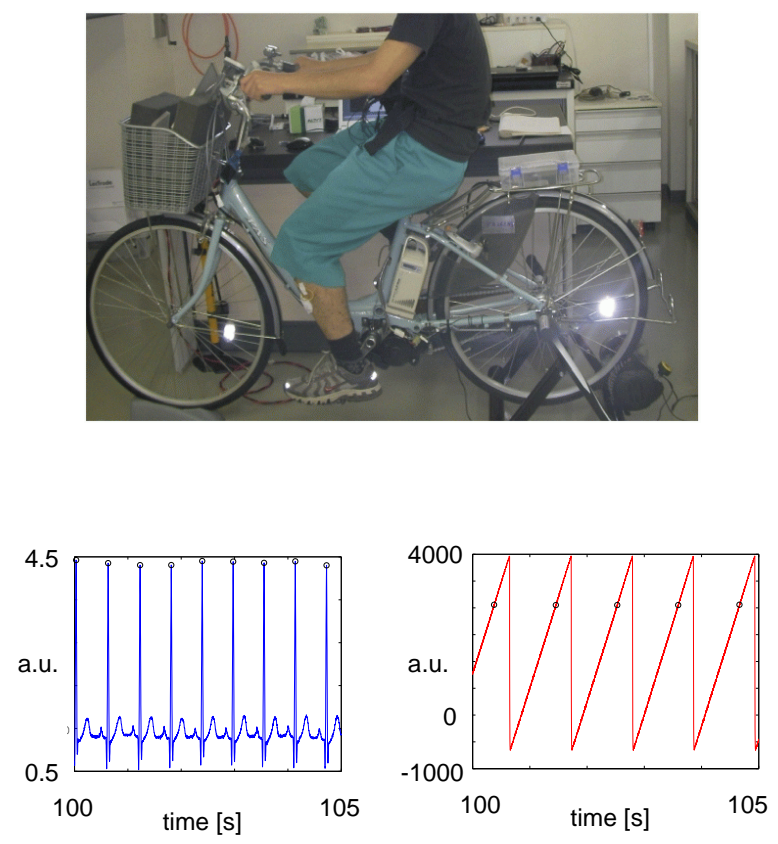

Figure 1: Top: the experimental environment. Bottom left: ECG. The circles represent the $\mathrm{R}$ peaks. Bottom right: crank signal. The circles represent the top dead centers (TDCs) for the right pedal.

We measured the electrocardiogram (ECG) and the crank signal. Both signals were digitized with a sampling rate of 1,000 Hz. A typical data are shown in Fig.1, bottom. The subject freely performed the exercises both with and without music. In the case without music, the averaged heart rate and cadence were $97.3( \pm 5.7) \mathrm{bpm}$ and 55.4 $( \pm 3.0) \mathrm{rpm}$, respectively. Meanwhile, in the case with music, those were $100.4( \pm 6.4) \mathrm{bpm}$ and $54.0( \pm 2.2) \mathrm{rpm}$, respectively. 

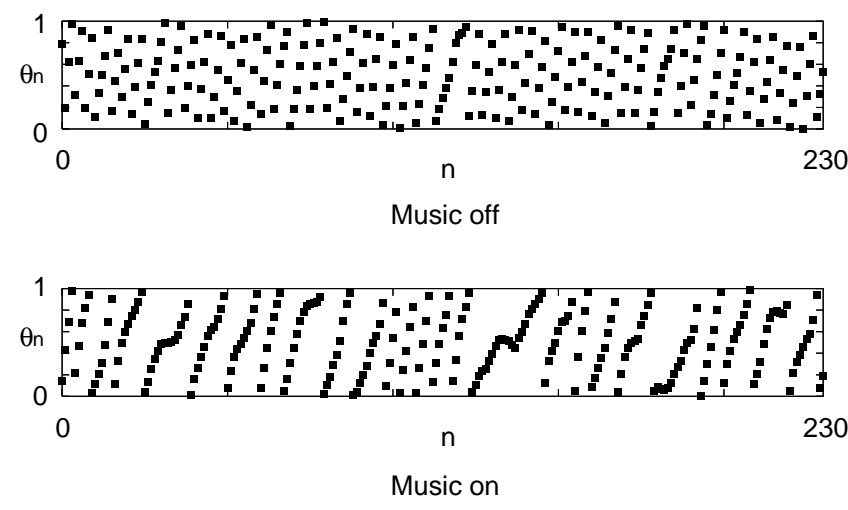

Figure 2: The time series of phase difference in the experiments. Top: a time series without music modulation. Bottom: a time series with music modulation.

The ratio of heart rate to cadence is roughly 2 to 1 in both conditions and there are, thus, two heart beats during one cycle of pedaling. However, the ratio of heart rate to cadence could change depending on subjects or experimental conditions. To extract rhythms, we define the phase difference $\theta_{n}$ as follows;

$$
\begin{aligned}
\theta_{n} & =\frac{R_{m}-P_{n}}{R_{m+1}-R_{m}}+0.5 \quad \text { if } \quad\left|R_{m}-P_{n}\right| \leq\left|P_{n}-R_{m+1}\right| \\
& =\frac{R_{m+1}-P_{n}}{R_{m+1}-R_{m}}+0.5 \quad \text { otherwise, }
\end{aligned}
$$

where $R_{m}$ and $P_{n}$ are respectively the time of appearance of the $m$-th R peak and the $n$-th top dead centers (TDC) for the right pedal, and $P_{n}$ satisfies $R_{m} \leq P_{n}<R_{m+1}$.

Only one heartbeat occurs during one cycle of pedaling with this normalization. The time series of phase difference with and without music are shown in Fig.2. With music modulation, stagnation motion around synchrony and the transitory synchronies between heartbeats and pedaling rhythms emerge, while only random phase drift is observed without music modulation.

\section{Random return maps from time series}

We construct return plots from time series of the phase difference $\theta_{n}$,. The return map without music modulation is modeled by the following random rotation map,

$$
\theta_{n+1}=\theta_{n}+\omega+\xi_{n}(\bmod 1),
$$

where the phase parameter $\omega \sim 0.22$ and the rotation number $\rho \sim 0.22$. Both are estimated by the experimental data. The random variable $\xi_{n}$ is an independent white Gaussian noise which follows $N\left(0,\left(0.05^{2}\right)\right)$. Random phase drift is observed in this model. The rotation number $\rho$, given as

$$
\rho=\lim _{n \rightarrow \infty} \frac{\sum_{k=1}^{n} \theta_{k}}{n}
$$

equals to $\omega$ in this case.

The return map with music modulation is given as the following random circle map,

$$
\theta_{n+1}=\theta_{n}+\omega^{\prime}+\xi_{n}-\eta_{n} \frac{K}{2 \pi} \cos \left(4 \pi \theta_{n}\right)(\bmod 1),
$$

where $\omega^{\prime}=0.15$, which is estimated by the experimental data. $K>0$ is a control parameter for the amplitude of external force. The model includes two random variables $\xi_{n}$ and $\eta_{n}$. The second random variable $\eta_{n}$ is an independent telegraphic noise whose amplitude is 0 with probability $p$ and 1 with probability $1-p$. The additive white Gaussian noise, $\xi_{n}$, is a model for the internal noise, which expresses a high dimensional dynamics within the body and the entire physiological system, and the multiplicative telegraphic noise, $\eta_{n}$, is those for the external noise, which corresponds to the music modulation which is with relatively smaller scale than the internal noise.

The mean rotation number $\rho^{\prime} \sim 0.13$ is estimated by the experimental data. The rotation numbers $\rho^{\prime}$ of the model (4) for $K \in[0,2]$, and $p \in[0,1]$, are numerically calculated and shown in Fig. (3). The line indicates $\rho^{\prime}=0.13$. We choose a parameter $(K, p)=(0.8,0.28)$ on the line, to fit the rotation number of the model to the estimated value, and to fit the shape of the random return map to the return plot of the experimental data.

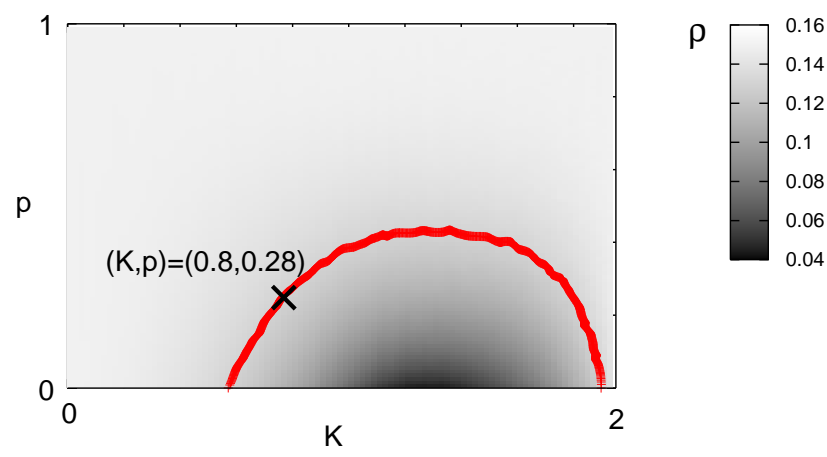

Figure 3: The rotation number $\rho^{\prime}$ of the equation (4) as a function of $K$ and $p$. The line corresponds to $\rho^{\prime}=0.13$. The selected parameter $(K, p)=(0.8,0.28)$ is denoted in the figure.

The constructed random return maps are shown in Fig.4. To get the graph, we set initial conditions and random realizations, numerically iterate each models with presence of the noise, and obtain return plots of the generated time series.

In order to check the validity of our model, we observe the time series and the invariant density generated by the model. The generated time series in an interval for 230 steps are depicted in Fig.5 and changes of the invariant densities are shown in Fig.6. One can see that the time series generated by the model qualitatively describes stagnation 

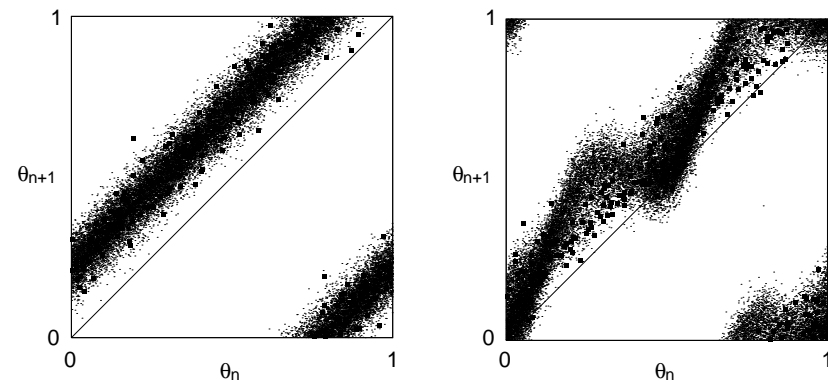

Figure 4: The random return maps generated by the model with $(K, p)=(0,0)$ and $(0.8,0.28)$ are shown with a set of scattered dots. The return plots of the experimental data are indicated with a set of smaller points. The left figure is those without music modulation and by the equation (2). The right figure is those with music modulation and by the equation (4).

motion in the experimental data. The invariant density generated by the model with music modulation is with two humps corresponding to each stagnation motion around synchrony.
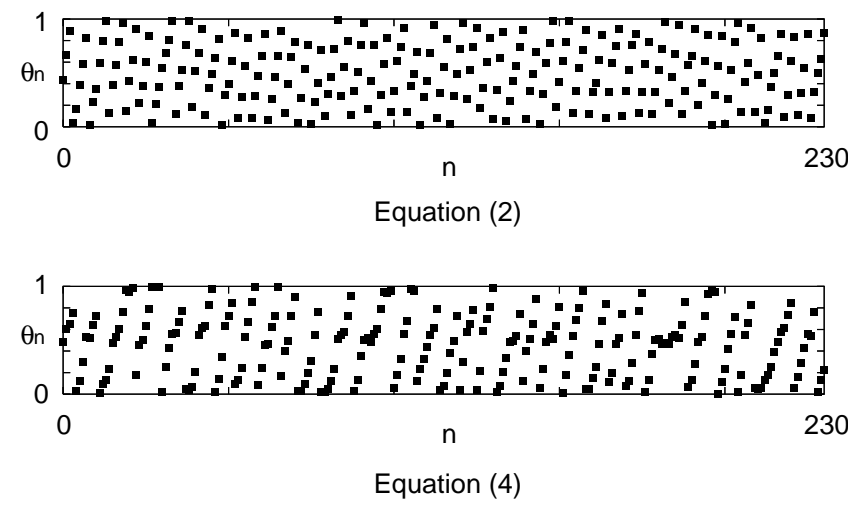

Figure 5: The time series of phase difference generated by the model. Top: a typical time series generated by the equation (2). Bottom: a typical time series generated by the equation (4).

\section{Summary}

We investigated the effect of music from the viewpoint of the interaction between human heartbeats and pedaling rhythms during the cycling exercise. We derived the phase difference between the heartbeats and the pedaling rhythms, and observed its time series. As a result, we found the transitory stagnation motion in the physiological rhythms with music modulation. We, then, constructed random return maps from the data. The dynamics without music is modeled by a random rotation map and the dynam-
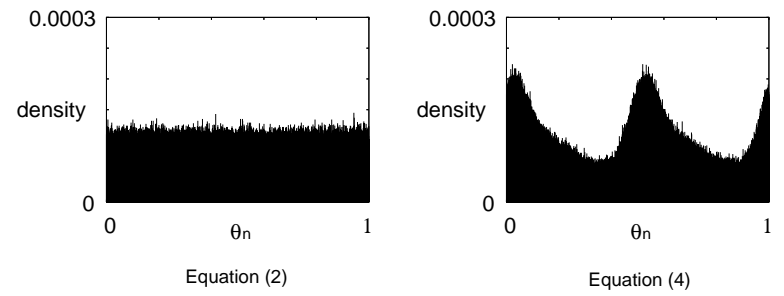

Figure 6: The invariant density generated by the model. Left: the density generated by the equation (2). Right: the density generated by the equation (4).

ics with music by a random circle map with two stochastic terms. The additive noise is the model of the internal noise, which expresses a high dimensional dynamics within the body, and the multiplicative noise is the model of external noise, which expresses the music modulation. The time series and the invariant density generated by our model qualitatively describe the phenomena observed in the experiments.

In conclusion, we consider that music changes the dynamical structure to induce the transition of synchrony and indirectly acts on involuntary rhythms. The effect of music is often discussed by using the averaged heart rate, but in our experiment there was no large difference of the averaged heart rates between dynamics with and without music modulation. The subject answered better mood under the condition with music in the introspective report, which indicates that there is an effect with music modulation. Our analyses capture them as transitory stagnation motion around synchrony in a random dynamics with two stochastic terms.

The presented random dynamical systems approaches, in general, may shed light on studies on finite scale phenomena in open non-autonomous dynamics, such as physiological rhythms modulated by one-time music listening that is studied in this paper, whose importance has been recognized in a wide range of scientific areas.

\section{Acknowledgments}

This work was supported by a collaboration grant form YAMAHA Motor Co., Ltd.

\section{References}

[1] M. Courtemanche, L. Glass, J. Bi Lair, D. Scaglioti'i, and D. Gordon, "A circle map in a human heart," Physica, D40, p299-310, (1989).

[2] L. Glass, M. R. Guevara, A. Shrier, R. Perez, "Bifurcation and Chaos in a Periodically Stimulated Cardiac Oscillator," Physica, 7D, p89-101, (1983). 
[3] L. Glass and M.C. Mackey, "From clocks to chaos: the rhythms of life," Princeton Univ. Press, Princeton, NJ, (1988).

[4] C. Schäfer, M. Rosenblum, H. Abel, and J. Kurths, "Synchronization in the human cardiorespiratory system," Phy. Rev., E60(1), p857-870, (1999).

[5] M. Fukumoto and S. Nomura, "The change in the synchronization between heartbeats and music," J. Medical Informatics $\mathcal{F}$ Technologies, 13, p229-234, (2009).

[6] K. Nomura, Y. Takei and Y. Yanagida, "Comparison of cardio-locomotor synchronization during running and cycling," Eur J Appl Physiol., 89(3-4, p221-229, (2003).

[7] W. Rejeski, "Perceived exertion: An active or passive process?,' Journal of Sport Psychology, 75, p371-378, (1985).

[8] G. Tenenbaumm, R. Lidor, N. Lavyan, K. Morrow, S. Tonnel, A. Gershgoren, J. Meis, and M. Johnson, "The effect of music type on running perseverance and coping with effort sensations," Psychology of Sport and Exercise, 5(2, p89-109, (2004).

[9] Y. Sato, M. Iima and Y. Tasaka, "Random dynamics from time-serieses of rotating fluid," Hokkaido University Preprint Series in Mathematics, 979 (2011), submitted. 